\title{
THE EMBEDDING PROBLEM \\ OVER A HILBERTIAN PAC-FIELD
}

\author{
Michael D. Fried*, UC Irvine \\ Helmut Völklein**, $U$ of Florida and Universität Erlangen
}

\begin{abstract}
We show that the absolute Galois group of a countable Hilbertian $\mathrm{P}$ (seudo)$\mathrm{A}$ (lgebraically) $\mathrm{C}($ losed) field of characteristic 0 is a free profinite group of countably infinite rank (Theorem A). As a consequence, $G(\bar{Q} / Q)$ is the extension of groups with a fairly simple structure (e.g., $\prod_{n=2}^{\infty} S_{n}$ ) by a countably free group. In addition, we characterize those PAC fields over which every finite group is a Galois group as those with the $R G$-Hilbertian property (Theorem B).
\end{abstract}

\section{INTRODUCTION}

All fields occurring in this paper are assumed to have characteristic 0 . A field $P$ is called $\mathrm{P}$ (seudo)A(lgebraically) $\mathrm{C}($ losed) if every absolutely irreducible variety defined over $P$ has a $P$-rational point. We use the methods of [FrVo] - to which this paper is a sequel - to prove a long-standing conjecture on Hilbertian PAC-fields $P$ : Every finite embedding problem over $P$ is solvable (Theorem A). For countable $P$ this, combined with a result of Iwasawa, implies that the absolute Galois group of $P$ is $\omega$-free; that is, $G(\bar{P} / P)$ is a free profinite group of countably infinite rank, denoted $\hat{F}_{\omega}$.

By a result of [FrJ; 2], every countable Hilbertian field $k$ has a Galois extension $P / k$ with $P$ Hilbertian and PAC, and $G(P / k) \cong \prod_{n=2}^{\infty} S_{n}$ (where $S_{n}$ is the symmetric group of degree $n$ ). From the above, $G(\bar{k} / P)=G(\bar{P} / P) \cong \hat{F}_{\omega}$, and we get the exact sequence

$$
1 \rightarrow \hat{F}_{\omega} \rightarrow G(\bar{k} / k) \rightarrow \prod_{n=2}^{\infty} S_{n} \rightarrow 1 .
$$

*Supported by NSA grant MDA 904-91-H-0057. and BSF grant \#87-00038

** Supported by NSA grant MDA 904-89-H-2028 
This holds in particular for $k$ the rational field $Q$ (or any algebraic number field). In this case it can be seen as a counterpart to Shafarevich's conjecture, which says that the abelian closure of $k$ has an $\omega$-free absolute Galois group: This would imply that $G(\bar{k} / k)$ is the extension of an abelian group by $\hat{F}_{\omega}$.

Now suppose that the countable Hilbertian field $k$ has in addition projective absolute Galois group. (This holds, for example, for the abelian closure of a number field.) Then $G(\bar{k} / k)$ is the semi-direct product of an $\omega$-free normal subgroup and a subgroup isomorphic to the universal frattini cover of the group $\prod_{n=2}^{\infty} S_{n}$ (Corollary 2).

In [FrVo] it was proved that each PAC-field $P$ of characteristic 0 has the following property: Every finite group is the Galois group of a regular extension $L / P(x)$, where $x$ is transcendental over $P$, and "regular" means - following common abuse - that $P$ is algebraically closed in $L$. In order to conclude that each finite group is a Galois group over $P$, it suffices to know that Hilbert's irreducibility theorem holds for regular Galois extensions of $P(x)$. This led to the concept of $R G$-Hilbertian: We define a field $P$ to be RG-Hilbertian if Hilbert's irreducibility theorem holds for regular Galois extensions of $P(x)$. We prove that a PAC-field $P$ of characteristic 0 is RG-Hilbertian if and only if every finite group is a Galois group over $P$ (Theorem B) - a parallel to our Theorem A, which says that a PAC-field $P$ of characteristic 0 is Hilbertian if and only if all finite embedding problems over $P$ are solvable. By example we demonstrate that the RG-Hilbertian property is actually weaker than the full Hilbertian property: the field $P$ can be chosen such that every finite group is a Galois group over $P$, but not every finite embedding problem over $P$ is solvable.

The main theme of the paper $[\mathrm{FrVo}]$ is to show that for a fixed finite group $G$ with trivial center, $G$ is the Galois group of a regular extension of $k(x)$, for some field $k$, if and only if there exist $k$-rational points on certain algebraic varieties. Here we use an extension of this, namely that also the solvability of certain embedding problems over $k$ is implied by the existence of $k$-rational points on certain varieties.

AMS Subject classification: 11G35, 12F10, 14D20, 14E20, 14G05, 20B25, 20C25

Keywords: Embedding problems; Galois groups; PAC-fields; Hilbertian fields; $\omega$-free profinite groups; $G(\bar{Q} / Q)$ as an extension. 
Comments on PAC fields: PAC-fields first appeared in $[\mathrm{Ax}]$. They have been studied since then by many authors (cf. [FrJ]). PAC fields have projective absolute Galois group - a result of Ax [FrJ; Theorem 10.17]. Conversely, if $H$ is a projective profinite group, then there exists a PAC field $P$ such that $H$ is the absolute Galois group of $P$-an observation of Lubotzky and van den Dries [LD]. New examples of Hilbertian PACfields (besides those constructed in [FrJ,2]) have recently been found by F. Pop. For example, one obtains such a field when adjoining $\sqrt{-1}$ to the field $Q_{r e}$ of all totally real algebraic numbers. Then our Theorem A implies:

$$
G\left(\bar{Q} / Q_{r e}(\sqrt{-1})\right) \cong \hat{F}_{\omega}
$$

On the other hand, the abelian closure of any number field has projective absolute Galois group, and it is Hilbertian [FrJ; Theorem 15.6]. But Frey noted that such a field isn't PAC ([Fy] or [FrJ; Corollary 10.15]). Shafarevich conjectured that the abelian closure of a number field has an $\omega$-free absolute Galois group. We know of no counterexample to the following:

Conjecture: If the absolute Galois group of a countable Hilbertian field is projective, then it is already $\omega$-free. 
Our proof of the corresponding result for PAC-fields would also prove this conjecture if one could show that each finite group $G$ satisfying the hypothesis of Lemma 2 has this property: One of the infinitely many absolutely irreducible $Q$-varieties associated to $G$ in [FrVo, Prop. 1] has a nonempty $Q$-subvariety that is unirational over $\bar{Q}$ (and therefore has a point over each field with projective absolute Galois group).

We remark that the following consequence of the above conjecture holds [FrJ; Thm. 24.50]: If $k$ is countable Hilbertian with projective absolute Galois group, then $G\left(k_{\mathrm{sol}} / k\right)$ is the free pro-solvable group of countably infinite rank, where $k_{\text {sol }}$ denotes the solvable closure of $k$. (Originally proved by Iwasawa [Iw] for $k$ the abelian closure of a number field.)

Notations: As above, all occurring fields are assumed to have characteristic 0 . The algebraic closure of a field $k$ is denoted by $\bar{k}$. The absolute Galois group $G(\bar{k} / k)$ of $k$ is denoted by $G_{k}$. In expressions like $k(x)$ or $P(x)$ always $x$ denotes an indeterminate, transcendental over the fields $k$ and $P$. The semi-direct product of groups $A$ and $B$ is written as $A \times{ }^{s} B$ (where $A$ is normal). The normalizer (resp., centralizer) of $A$ in $B$ is denoted $N_{B}(A)$ (resp., $C_{B}(A)$ ). Finally, $\operatorname{Aut}(A)$ (resp., $\operatorname{Inn}(A)$ ) is the automorphism group of $A$ (resp., the group of inner automorphisms of $A$ ). Other notations as introduced above. 


\section{THE EMBEDDING PROBLEM OVER A HILBERTIAN PAC- FIELD}

We are going to show that all finite embedding problems over a Hilbertian PAC-field $P$ are solvable (Theorem A). Our first lemma is a geometric form of the "field crossing argument" from [FrJ; §23.1]; the same idea occurs also in [Se, 2.1].

Lemma 1: Let $\mathcal{H}^{\prime} \rightarrow \mathcal{H}$ be an unramified Galois cover of absolutely irreducible varieties defined over a $P A C$-field $P$ of characteristic 0 . Assume all automorphisms of the cover are defined over $P$. If $P^{\prime} / P$ is any Galois extension (inside a fixed algebraic closure of $P$ ) with Galois group isomorphic to a subgroup $F$ of $\operatorname{Aut}\left(\mathcal{H}^{\prime} / \mathcal{H}\right)$, then there exists a $P$-rational point $\boldsymbol{p}$ of $\mathcal{H}$ and a point $\boldsymbol{p}^{\prime} \in \mathcal{H}^{\prime}$ lying over $\boldsymbol{p}$ with the following property: $P\left(\boldsymbol{p}^{\prime}\right)=P^{\prime}$, and the $G_{P}$-orbit of $\boldsymbol{p}^{\prime}$ coincides with the $F$-orbit of $\boldsymbol{p}^{\prime}$.

Proof: By hypothesis there is a homomorphism $\beta: G_{P} \rightarrow \operatorname{Aut}\left(\mathcal{H}^{\prime} / \mathcal{H}\right)$ with kernel $G\left(\bar{P} / P^{\prime}\right)$ and with image $F$. Since $G_{P}$ acts trivially on $\operatorname{Aut}\left(\mathcal{H}^{\prime} / \mathcal{H}\right)$, we can view $\beta$ as a 1 -cocycle of $G_{P}$ with values in $\operatorname{Aut}\left(\mathcal{H}^{\prime}\right)$. By Weil's cocycle criterion [W], such a cocycle corresponds to a twisted form of $\mathcal{H}^{\prime}$ over $P$. We identify the $\bar{P}$-points of the twisted form and of the original variety $\mathcal{H}^{\prime}$. Then the twisted form defines a new action of $G_{P}$ on these $\bar{P}$-points $\boldsymbol{p}^{\prime}$ : If we denote the old action of $g \in G_{P}$ by $\boldsymbol{p}^{\prime} \mapsto g \boldsymbol{p}^{\prime}$, then the new action of $g$ sends $\boldsymbol{p}^{\prime}$ to $g \beta(g) \boldsymbol{p}^{\prime}$.

As $P$ is PAC, there is a point $\boldsymbol{p}^{\prime} \in \mathcal{H}^{\prime}$ that is rational over $P$ with respect to the twisted form. This means that $\beta(g) \boldsymbol{p}^{\prime}=g^{-1} \boldsymbol{p}^{\prime}$ for each $g \in G_{P}$. Thus $\operatorname{ker}(\beta)$ is the stabilizer of $\boldsymbol{p}^{\prime}$ in $G_{P}$. Hence $P\left(\boldsymbol{p}^{\prime}\right)=P^{\prime}$.

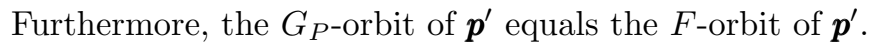


In Lemma 2 and 3 we assume $P$ is a Hilbertian PAC-field (of characteristic 0). Lemma 2 invokes the main results of $[\mathrm{FrVo}]$; this is the heart of the proof of Theorem A. The hypothesis on the Schur multiplier comes in because our application of $[\mathrm{FrVo}]$ is based on the Conway-Parker theorem (cf. [FrVo, §2.2]) which requires this hypothesis.

Lemma 2: Let $H$ be a finite group and $G$ a normal subgroup of $H$, such that $C_{H}(G)=\{1\}$. Assume the Schur multiplier of $G$ is trivial. Then each Galois extension $P^{\prime} / P$ with Galois group isomorphic to $H / G$ can be embedded in a Galois extension $P^{\prime \prime} / P$ for which there is an isomorphism $G\left(P^{\prime \prime} / P\right) \rightarrow H$ sending $G\left(P^{\prime \prime} / P^{\prime}\right)$ to $G$.

Proof: By [FrVo, Proposition 3], under the given hypotheses on $G$ there exists an unramified Galois cover $\mathcal{H}^{\prime} \rightarrow \mathcal{H}$ of absolutely irreducible varieties defined over $Q$, and an identification of the automorphism group $\operatorname{Aut}\left(\mathcal{H}^{\prime} / \mathcal{H}\right)$ of this cover with the group $\operatorname{Out}(G)=\operatorname{Aut}(G) / \operatorname{Inn}(G)$, such that the following hold. First: all automorphisms of the cover $\mathcal{H}^{\prime} \rightarrow \mathcal{H}$ are defined over $Q$. Furthermore, for each point $\boldsymbol{p} \in \mathcal{H}$, rational over some field $k$, and for each point $\boldsymbol{p}^{\prime} \in \mathcal{H}^{\prime}$ lying over $\boldsymbol{p}$, there is a regular extension $L / k^{\prime}(x)$, where $k^{\prime}=k\left(\boldsymbol{p}^{\prime}\right)$, with the following properties: $L$ is Galois over $k(x)$, and the group $G(L / k(x))$ is isomorphic to the group of all $g \in \operatorname{Aut}(G)$ for which the image of $g$ in $\operatorname{Out}(G)=\operatorname{Aut}\left(\mathcal{H}^{\prime} / \mathcal{H}\right)$ maps $\boldsymbol{p}^{\prime}$ to a $G\left(k^{\prime} / k\right)$-conjugate of $\boldsymbol{p}^{\prime}$. Under this isomorphism, the subgroup $G\left(L / k^{\prime}(x)\right)$ is mapped onto $\operatorname{Inn}(G)$.

Now assume $k=P$ is a Hilbertian PAC-field, and consider the given Galois extension $P^{\prime} / P$ with $G\left(P^{\prime} / P\right) \cong H / G$. Since $C_{H}(G)=\{1\}$, the conjugation action of $H$ on $G$ induces an isomorphism from $H$ to a subgroup $\bar{H}$ of $\operatorname{Aut}(G)$. Hence $G\left(P^{\prime} / P\right) \cong H / G \cong \bar{H} / \operatorname{Inn}(G)$, and $F \stackrel{\text { def }}{=} \bar{H} / \operatorname{Inn}(G)$ is a subgroup of $\operatorname{Out}(G)=\operatorname{Aut}\left(\mathcal{H}^{\prime} / \mathcal{H}\right)$. Thus by Lemma 1 we may choose $\boldsymbol{p}$ and $\boldsymbol{p}^{\prime}$ so that $P\left(\boldsymbol{p}^{\prime}\right)=P^{\prime}$, and the $G\left(P^{\prime} / P\right)$ orbit of $\boldsymbol{p}^{\prime}$ equals the $F$-orbit of $\boldsymbol{p}^{\prime}$. For the associated Galois extension $L / P(x)$, it follows that $G(L / P(x))$ is isomorphic to the group of all $g \in \operatorname{Aut}(G)$ for which the image of $g$ in $\operatorname{Out}(G)$ lies in $F$. But this group is just $\bar{H}$. Thus $G(L / P(x))$ is isomorphic to $\bar{H}$, under an isomorphism that identifies $G\left(L / P^{\prime}(x)\right)$ with $\operatorname{Inn}(G)$. Hence $G(L / P(x))$ is isomorphic to $H$, under an isomorphism that maps $G\left(L / P^{\prime}(x)\right)$ to $G$.

Since $P$ is Hilbertian, we can specialize $x$ to get an extension $P^{\prime \prime} / P$ which is still Galois with Galois group isomorphic to $H$, and this isomorphism identifies $G\left(P^{\prime \prime} / P^{\prime}\right)$ with $G$ (cf. [FrJ, Lemma 12.12]). 
Now we are ready to tackle the general embedding problem over $P$. The projectivity of $G_{P}$ allows us to reduce to the case of split embedding problems, and these are reduced group-theoretically to the special case of Lemma 2. Only then does the PAC-assumption on $P$ come into play.

Lemma 3: Let $A$ be any finite group, and $B$ a normal subgroup. Then each Galois extension $P^{\prime} / P$ with Galois group isomorphic to $A / B$ can be embedded in a Galois extension $P^{\prime \prime} / P$ for which there is an isomorphism $G\left(P^{\prime \prime} / P\right) \rightarrow A$ sending $G\left(P^{\prime \prime} / P^{\prime}\right)$ to $B$.

Proof: By induction on the order of $B$ we may assume that $B$ is a minimal normal subgroup of $A$. Thus $B \cong S^{m}$, the direct product of $m$ copies of a simple group $S$. The remainder of the proof falls into two parts. The first observes that we may assume that $A$ splits over $B$ (a version of "Jarden's Lemma" from [Ma, p. 231]).

Part 1: Application of the projectivity of $G_{P}$. Since $G_{P}$ is projective there exists $\alpha: G_{P} \rightarrow A$ such that the composition of $\alpha$ with the natural map from $A$ to $A / B$ has kernel $G_{P^{\prime}}$ (see [FrJ, Th. 10.17]). This composition is surjective, so the image $C_{1}$ of $\alpha$ satisfies $A=B C_{1}$. Then $A$ is a homomorphic image of the outer semi-direct product $A_{1}=B \times{ }^{s} C_{1}$, under the natural map $\pi$ that sends $(b, c)$ to $b c$. Suppose that the lemma holds with $A_{1}$ in place of $A$ and for the fixed field $P_{1}^{\prime}$ of $\operatorname{ker}(\alpha)$ in place of $P^{\prime}$ (but for the same $B$ ). Then we may embed $P_{1}^{\prime}$ in an extension $P_{1}^{\prime \prime}$ with group $G\left(P_{1}^{\prime \prime} / P\right) \cong A_{1}$, such that $G\left(P_{1}^{\prime \prime} / P_{1}^{\prime}\right)$ corresponds to $B$. The fixed field of $\operatorname{ker}(\pi)$ in $P_{1}^{\prime \prime}$ is the desired $P^{\prime \prime}$. This shows that it suffices to consider split extensions with kernel $B$.

Part 2: Reduction to the special case of Lemma 2. From now on assume $A=B \times{ }^{s} C$, for some $C$. Every finite split embedding problem with abelian kernel over a Hilbertian field is solvable ([Ma; Folg. 1, p. 231] or [FrJ; Thm. 24.50]). Thus we may further assume that $S$ is non-abelian (simple). Then $B=S^{m}$ is perfect, and so it has a universal central extension $\tilde{B}$. Furthermore, $\tilde{B}$ has trivial Schur multiplier (see [Ka; p. 152153]). By the universal property of the universal central extension, the action of $C$ on $B$ lifts uniquely to an action of $C$ on $\tilde{B}$. Form accordingly the semi-direct product $\hat{A}=\tilde{B} \times{ }^{s} C$.

Let $T$ be a non-abelian finite simple group with trivial Schur multiplier (e.g., $T=\mathrm{SL}_{2}(8)$ [Hu, Satz 25.7]). Consider the regular wreath product $H$ of $\hat{A}$ with $T$ (e.g., [Hu, Def. 15.6]). Thus $H=T^{j} \times^{s} \hat{A}$, with $j=|\hat{A}|$, and $\hat{A}$ acts on $T^{j}$ by permuting the factors in its regular permutation representation. Then $H=\left(T^{j} \times{ }^{s} \tilde{B}\right) \times{ }^{s} C=G \times{ }^{s} C$, with $G=T^{j} \times{ }^{s} \tilde{B}$. Clearly, $C_{H}\left(T^{j}\right)=\{1\}$, hence also $C_{H}(G)=\{1\}$. 
Since $T$ and $\tilde{B}$ are perfect groups with trivial Schur multiplier, every central extension of these groups splits. From this one concludes easily that also every central extension of $G=T^{j} \times{ }^{s} \tilde{B}$ splits. Thus also $G$ has trivial Schur multiplier.

We have now shown that $G$ and $H$ satisfy the hypotheses of Lemma 2. It follows that the given Galois extension $P^{\prime} / P$ with group isomorphic to $A / B \cong H / G$ can be embedded in an extension $K / P$ with $G(K / P) \cong H$, such that $G\left(K / P^{\prime}\right)$ corresponds to $G$. Then the fixed field in $K$ of the kernel of the natural map from $H$ onto $A$ (which sends $G$ onto $B$ ) is the desired $P^{\prime \prime}$. $\quad$ 口

Lemma 4: For every surjection $h: E \rightarrow C$ of finite groups there exists a surjection $g: A \rightarrow E$ of finite groups such that every automorphism $\gamma$ of $C$ lifts to an automorphism $\alpha$ of $A: h \circ g \circ \alpha=\gamma \circ h \circ g$.

Proof: Let $H$ be the semi-direct product of $C$ with its automorphism group $\operatorname{Aut}(C)$. Choose a surjection $\mathcal{F} \rightarrow H$ with $\mathcal{F}$ a free group (of finite rank), and let $\mathcal{F}_{0}$ be the inverse image of $C$ in $\mathcal{F}$. Then $\mathcal{F}_{0}$ is again a free group, of rank greater or equal to that of $\mathcal{F}$ (by Schreier's subgroup formula; e.g. [FrJ; Prop. 15.25]). Thus by choosing $\mathcal{F}$ of suitably large rank we can assure that the map $\mathcal{F}_{0} \rightarrow C$ can be factored as $h \circ f$ for some surjection $f: \mathcal{F}_{0} \rightarrow E$. Let $\mathcal{R}_{0}$ be the intersection of all $\mathcal{F}$-conjugates of the kernel of $f$.

Every automorphism $\gamma$ of $C$ is induced from an inner automorphism of $H$, hence from an inner automorphism of $\mathcal{F}$, and thus from an inner automorphism of the finite group $\mathcal{F} / \mathcal{R}_{0}$. This inner automorphism restricts to an automorphism of $A=\mathcal{F}_{0} / \mathcal{R}_{0}$ that still induces $\gamma$. By construction, the natural map $A \rightarrow C$ factors as $h \circ g$ for some surjection $g: A \rightarrow E . \quad \square$

One says that all finite embedding problems over a field $P$ are solvable if for every surjection $h: E \rightarrow C$ of finite groups and for every surjection $\lambda: G_{P} \rightarrow C$ there exists a surjection $\epsilon: G_{P} \rightarrow E$ with $h \circ \epsilon=\lambda$. Recall that a profinite group is called $\omega$-free if it is isomorphic to the free profinite group $\hat{F}_{\omega}$ of countably infinite rank $[\mathrm{FrJ}, \S 15.5]$.

Theorem A: A PAC-field $P$ of characteristic 0 is Hilbertian if and only if all finite embedding problems over $P$ are solvable. In particular, a countable PAC-field of characteristic 0 is Hilbertian if and only if its absolute Galois group is $\omega$-free.

Proof: By a result of Iwasawa [Iw, p. 567] (see [FrJ; Cor. 24.2 and Ex. 15.13(b)]), it suffices to prove the first assertion. The "if" part is a result of Roquette [FrJ; Cor. 24.38]; we give a new proof in Lemma 5 below, using the methods of this paper. 
Now assume $P$ is Hilbertian. We have to show that all finite embedding problems over $P$ are solvable. So suppose we have a surjection $h: E \rightarrow C$ of finite groups and a surjection $\lambda: G_{P} \rightarrow C$. Let $g: A \rightarrow E$ be as in Lemma 4. It follows from Lemma 3 that there is a surjection $\theta: G_{P} \rightarrow A$ with $\operatorname{ker}(h \circ g \circ \theta)=\operatorname{ker}(\lambda)$. Thus $\gamma \circ h \circ g \circ \theta=\lambda$ for some automorphism $\gamma$ of $C$. By choice of $A$, we can lift $\gamma$ to an automorphism $\alpha$ of $A$. Then $\epsilon \stackrel{\text { def }}{=} g \circ \alpha \circ \theta$ is a surjection $G_{P} \rightarrow E$ with $h \epsilon=h \circ g \circ \alpha \circ \theta=\gamma \circ h \circ g \circ \theta=\lambda$, as desired.

By a result of [FrJ, 2] (c.f. [FrJ; Th. 16.46]), every countable Hilbertian field $k$ has a Galois extension $P / k$ with $P$ Hilbertian and PAC, and $G(P / k) \cong \prod_{i=1}^{\infty} S_{n_{i}}$. In the above references, there are certain special conditions on the sequence $\left(n_{i}\right)$, but the construction can easily be modified to yield $n_{i}=i$. For the convenience of the reader, we sketch the argument in Remark 1 below. From the Theorem we get $G_{P} \cong \hat{F}_{\omega}$.

Corollary 1: Suppose $k$ is a countable Hilbertian field of characteristic 0 . Then there is an exact sequence

$$
1 \rightarrow \hat{F}_{\omega} \rightarrow G_{k} \rightarrow \prod_{n=2}^{\infty} S_{n} \rightarrow 1
$$

The field $P$ with $G(P / k) \cong \prod_{n=2}^{\infty} S_{n}$ has the nice property that it is a rather small extension of $k$ with $\omega$-free absolute Galois group $G_{P}$. Its construction, however, is not canonical (see Remark 1 below). In particular, $G_{P}$ is not necessarily a characteristic subgroup of $G_{k}$. To have a more canonical example, consider the composite of all $S_{n^{-}}$extensions of $k$, for all $n$. This yields an analog to the consideration of $Q_{\mathrm{ab}}$ (the composite of all abelian extensions of $Q$ ) in Shafarevich's conjecture (Introduction). Actually, not much is changed if one excludes any finite number of values of $n$ in the above. For simplicity, we consider the composite $K$ of all $S_{n}$ - extensions of $k$ with $n \geq 5$. Remark 1 shows that $K$ is PAC. In the next paragraph we show that $K$ is also Hilbertian. Therefore, from Theorem A, $G_{K}$ is $\omega$-free.

By definition of $K$, the group $\Gamma=G(K / k)$ embeds as a subgroup of the product of (countably many) symmetric groups $S_{n_{i}}$, and projection from $\Gamma$ to any one of the factors is surjective (i.e., $\Gamma$ is a subdirect product of the $S_{n_{i}}$ ). Thus the closure $\Gamma^{\prime}$ of the commutator subgroup of $\Gamma$ is a subdirect product of the alternating groups $A_{n_{i}}$. As $n_{i} \geq 5$ these groups $A_{n_{i}}$ are simple. Hence $\Gamma^{\prime} \cong \prod A_{m_{j}}$ for some subsequence $\left(m_{j}\right)$ of $\left(n_{i}\right)$ (see [M; Lemma 1.3]; in fact $\Gamma^{\prime}$ is the product of countably many copies of $\prod_{n=5}^{\infty} A_{n}$ ). The fixed field of $\Gamma^{\prime}$ in $K$ is Hilbertian since it is an abelian extension of the Hilbertian field $k$ [FrJ; Thm. 15.6]. Thus $K$ is Hilbertian by Weissauer's theorem (see Remark 2 below) because $\Gamma^{\prime}$ has non-trivial finite normal subgroups.

Question: Is the composite of all $A_{n}$-extensions of $k$ also PAC? 
Remark 1: Construction of Hilbertian PAC-fields, after [FrJ, 2]. Let $k$ be countable and Hilbertian. An algebraic extension $P$ of $k$ is PAC if every absolutely irreducible projective curve defined over $k$ has infinitely many P-rational points [FrJ; Th. 10.4]. By a result of Lefschetz (see [FrJ, 2]) one can restrict to plane curves having only singularities of multiplicity 2 . There are only countably many pairs $(C, M)$ where $C$ is an absolutely irreducible projective plane curve defined over $k$ that has only singularities of multiplicity 2 , and $M$ is a finite set of $\bar{k}$-points of $C$. Enumerate these pairs as $\left(C_{1}, M_{1}\right),\left(C_{2}, M_{2}\right),\left(C_{3}, M_{3}\right), \ldots$ We are going to construct a Galois extension $P$ of $k$ such that each $C_{i}$ has a $P$-rational point not in $M_{i}$. Then $C_{i}$ has infinitely many $P$-rational points, and $P$ is PAC by the above.

For each $C_{i}$ there exists a point $O$ in the plane such that projection from $O$ to a suitable line yields a cover $\varphi_{i}: C_{i} \rightarrow \mathcal{P}^{1}$ (where $\mathcal{P}^{1}$ is the projective line) with the following properties: $\varphi_{i}$ is defined over $k$, and the Galois closure of the corresponding function field extension $k\left(C_{i}\right) / k(x)$ has Galois group $S_{n_{i}}$, where $n_{i}$ is the degree of the plane curve $C_{i}[\mathrm{FrJ}, 2]$. Since $k$ is Hilbertian, there exist infinitely many points $c \in C_{i}$ such that the Galois closure of the extension $k(c) / k$ also has group $S_{n_{i}}$; additionally, one can require that this Galois closure is linearly disjoint from any given finite extension of $k$ (see e.g., [Se, Prop. 4.10]).

Now we define recursively a sequence $\left(k_{i}\right)$ of linearly disjoint Galois extensions of $k$ and a strictly increasing sequence $\left(m_{i}\right)$ of integers with $G\left(k_{i} / k\right) \cong S_{m_{i}}$, such that $C_{i}$ has a $k_{i}$ - rational point not in $M_{i}$. Set $k_{0}=k, m_{0}=1$. To construct $k_{i}$ and $m_{i}$ for $i \geq 1$ choose an integer $n$ such that $m_{i} \stackrel{\text { def }}{=} n n_{i}>m_{i-1}$ (where again $n_{i}$ is the degree of $C_{i}$ ). A "sufficiently general" substitution of degree $n$ (with coefficients from $k$ ) in the equation defining $C_{i}$ yields a curve $\bar{C}_{i}$ of degree $m_{i}=n n_{i}$ that is again among the curves $C_{1}, C_{2}, \ldots$ By the preceding paragraph, there exists $c \in \bar{C}_{i}$ such that the natural map $\bar{C}_{i} \rightarrow C_{i}$ is defined at $c$ and does not map $c$ into $M_{i}$, the Galois closure $k_{i}$ of the extension $k(c) / k$ has group $S_{m_{i}}$ and $k_{i}$ is linearly disjoint from the composite $k_{0} \cdots k_{i-1}$. This concludes the construction of the above sequence $\left(k_{i}\right)$. Finally, using [FrJ; Lemma 15.8] one can "fill in" more fields during this inductive construction to get a sequence $\left(K_{j}\right)$ with $k_{i}=K_{m_{i}}$ and $G\left(K_{j} / k\right) \cong S_{j}$, such that for all $j$, the field $K_{j}$ is linearly disjoint from $K_{1} \cdots K_{j-1}$. Then the composite $P$ of all $K_{j}$ satisfies $G(P / k) \cong \prod_{j=2}^{\infty} S_{j}$. Further, $P$ is PAC because every curve $C_{i}$ has a $P$-rational point, and $P$ is Hilbertian by Weissauer's theorem (see Remark 2 below) because $G(P / k)$ has non-trivial finite normal subgroups. $\quad$ 口

The above shows that the composite $P_{N}$ of the fields $K_{j}$ with $j \geq N$ is still PAC and Hilbertian. Since the intersection of all these fields $P_{N}$ is just $k$, it follows that $G_{k}$ is the closure of an ascending union of $\omega$-free normal subgroups. 
Now we return to the set-up of Corollary 1. Assume additionally that $G_{k}$ is projective. This implies that the epimorphism $\varphi: G_{k} \rightarrow \prod_{n=2}^{\infty} S_{n}$ lifts to a homomorphism $\psi: G_{k} \rightarrow \mathcal{E}$, where $\mathcal{E}$ is the universal frattini cover of $\prod_{n=2}^{\infty} S_{n}$ : This is the minimal projective cover of $\prod_{n=2}^{\infty} S_{n}$, and the map $\mathcal{E} \rightarrow \prod_{n=2}^{\infty} S_{n}$ has pro-nilpotent kernel $\mathcal{N}$ (see [FrJ; Lemma 20.2, Prop. 20.33]). The map $\psi$ is surjective by the defining property of a frattini cover $[\mathrm{FrJ} ; \S 20.6]$. Further, $\operatorname{ker}(\psi)$ is normal in $\operatorname{ker}(\varphi) \cong \hat{F}_{\omega}$, and the quotient is isomorphic to the pro-nilpotent group $\mathcal{N}$. Hence also $\operatorname{ker}(\psi)$ is $\omega$-free (by Corollary 3 below). Finally, the map $\psi$ is a splitting extension since $\mathcal{E}$ is projective. Thus we have proved:

Corollary 2: Suppose $k$ is a countable Hilbertian field of characteristic 0 , and $G_{k}$ is projective. Then $G_{k}$ is the semi-direct product of an $\omega$-free normal subgroup and a subgroup isomorphic to the universal frattini cover of $\prod_{n=2}^{\infty} S_{n}$.

We recall that according to our conjecture from the Introduction, the group $G_{k}$ should itself be $\omega$-free in the situation of Corollary 2. Furthermore, as a consequence of the (folklore) conjecture that every finite group is the Galois group of a regular extension of $Q(x)$, every finite group should be a quotient of $G_{k}$ in the situation of Corollary 1.

Remark 2: Hilbertian fields versus subgroups of $\hat{F}_{\omega}$. Theorem A implies a "transfer theorem" that turns results about algebraic extensions of Hilbertian fields into results about subgroups of $\hat{F}_{\omega}$ (as noted by Jarden and Lubotzky $[\mathrm{JaLu}]$ ). Namely, consider a countable Hilbertian PAC-field $P$ (as above). Then $G_{P} \cong \hat{F}_{\omega}$ by Theorem A.

Now take a result saying that certain algebraic extensions of a Hilbertian field are again Hilbertian; e.g., Weissauer's theorem says that any non-trivial finite extension of a Galois extension of a Hilbertian field is again Hilbertian (see [Ws] for a non-standard proof and [Fr] for a standard proof). Theorem A then implies that certain analogously defined subgroups of an $\omega$-free group are again $\omega$-free. For example, Weissauer's theorem translates into the result that any proper closed subgroup $U_{1}$ of finite index in a normal subgroup $U$ of $\hat{F}_{\omega}$ is again $\omega$-free (a direct proof of this was given by Lubotzky-Melnikov-v. d. Dries; see [FrJ; Theorem 24.7]). Namely, the fixed field $P_{1}$ in $\bar{P}$ of $U_{1}$ is Hilbertian by Weissauer's theorem. Since every algebraic extension of a PAC-field is again PAC [FrJ; Cor. 10.7], it follows from Theorem A that $U_{1}=G_{P_{1}}$ is $\omega$-free.

There are several results about extensions of Hilbertian fields similar to Weissauer's (see [JaLu]). For most of them, the corresponding result about subgroups of $\hat{F}_{\omega}$ has been proved directly. However, this is not true for the result of Uchida [U] on pro-nilpotent extensions of Hilbertian fields, which translates into a new result: 
Corollary 3: If $N$ is a normal subgroup of $\hat{F}_{\omega}$ such that $\hat{F}_{\omega} / N$ is pro-nilpotent, and $U$ is a subgroup of $\hat{F}_{\omega}$ containing $N$, then $U$ is again $\omega$-free provided the index $\left[\hat{F}_{\omega}: U\right]$ is divisible by at least two distinct primes (in the sense of super-natural numbers).

A slightly different proof of this corollary is given in [JaLu], using [FrVo, Th. 2] instead of Theorem A.

\section{THE RG-HILBERTIAN PROPERTY}

The Hilbertian property can be rephrased as follows in terms of Galois extensions: A field $P$ is Hilbertian if and only if every finite Galois extension of $P(x)$ can be specialized to a Galois extension of $P$ with the same Galois group. In most applications (e.g., to the Inverse Galois Problem) it isn't the full Hilbertian property for $P$ that is used, but rather a weaker version: if $G$ is the Galois group of a regular extension of $P(x)$, then $G$ is also a Galois group over $P$. We formalize this.

Definition: We say a field $P$ is $R$ (egular)G(alois)- Hilbertian if every regular (finite) Galois extension of $P(x)$ can be specialized to a Galois extension of $P$ with the same Galois group.

If $P$ is a PAC-field of characteristic 0, then every finite group is the Galois group of a regular extension of $P(x)$, by [FrVo, Theorem 2]. Thus if $P$ is also RG-Hilbertian, then each finite group is a Galois group over $P$. The converse is also true:

Theorem B: A PAC-field $P$ of characteristic 0 is RG-Hilbertian if and only if every finite group is a Galois group over $P$.

It remains to prove the "if" part of Theorem B. This is a simple application of Lemma 1. We refine the argument a little to simultaneously give a new proof of the "if" part of Theorem A (originally due to Roquette). 
Lemma 5: Let $P$ be a $P A C$-field, and let $L / P(x)$ be a finite Galois extension. Assume that either:

(A) all finite embedding problems over $P$ are solvable; or

(B) $P$ is algebraically closed in $L$ and each finite group is a Galois group over $P$.

Then the extension $L / P(x)$ can be specialized to a Galois extension of $P$ with the same Galois group.

Proof: There is a non-singular curve $\Gamma$ defined and irreducible over $P$ with function field $L$. The extension $L / P(x)$ corresponds to a cover $\varphi: \Gamma \rightarrow \mathcal{P}^{1}$ defined over $P$. We identify the group $H=G(L / P(x))$ canonically with the automorphism group of this cover. The absolutely irreducible components $\Gamma_{1}, \ldots, \Gamma_{s}$ of $\Gamma$ are defined over the algebraic closure $P_{1}$ of $P$ in $L$, and $G=G\left(L / P_{1}(x)\right)$ is the subgroup of $H$ stabilizing $\Gamma_{1}, \ldots, \Gamma_{s}$. The group $H / G$ permutes $\Gamma_{1}, \ldots, \Gamma_{s}$ sharply transitively.

Both $H$ and $G_{P}$ act naturally on the $\bar{P}$-points of $\Gamma$. Since the automorphisms from $H$ are defined over $P$, the group $G_{P}$ centralizes $H$. The groups $H / G$ and $G_{P} / G_{P_{1}} \cong G\left(P_{1} / P\right)$ act sharply transitively on $\Gamma_{1}, \ldots, \Gamma_{s}$, and they centralize each other. Thus there exists an isomorphism $\bar{\alpha}: G_{P} / G_{P_{1}} \rightarrow H / G$ such that $\bar{g} \bar{\alpha}(\bar{g})$ fixes $\Gamma_{1}$ for each $\bar{g} \in G_{P} / G_{P_{1}}$. This yields a surjection $\alpha: G_{P} \rightarrow H / G$ such that $g \alpha(g)$ fixes $\Gamma_{1}$ for each $g \in G_{P}$.

Now let $\beta: G_{P} \rightarrow H$ be a surjection such that $\beta$ composed with the natural map $H \rightarrow H / G$ equals $\alpha$. Such $\beta$ exists in case (A) by the definition of an embedding problem, and it exists in case (B) because there $H=G$, and $H$ is a quotient of $G_{P}$.

As in the proof of Lemma 1 we view $\beta$ as a 1-cocycle of $G_{P}$ in $\operatorname{Aut}(\Gamma)$. Hence $\beta$ defines a twisted form of $\Gamma$, such that each $g \in G_{P}$ acts via this twisted form as $g \beta(g)$ on the $\bar{P}$-points of $\Gamma$ (cf. the proof of Lemma 1). Since $g \beta(g)$ fixes $\Gamma_{1}$ (because of the corresponding property of $\alpha$ ), it follows that $\Gamma_{1}$ is defined over $P$ in the twisted form. By the PAC property, $\Gamma_{1}$ has a $P$-rational point $c$ relative to the twisted form that does not lie over a branch point of $\varphi$. Now, as in Lemma $1, \varphi(c)$ is a $P$-rational point of $\mathcal{P}^{1}$ which yields the desired specialization $P(c) / P$ of the extension $L / P(x)$ with the same Galois group $H$. 口

Remark 3: By Theorems A and B, for a PAC-field $P$ the RG-Hilbertian and Hilbertian properties are equivalent to purely group-theoretic properties of the absolute Galois group $G_{P}: P$ is RG-Hilbertian if and only if each finite group is a quotient of $G_{P}$; and $P$ is Hilbertian if and only if each finite embedding problem for $G_{P}$ is solvable.

We conclude with an example showing that the RG-Hilbertian property is actually weaker than the full Hilbertian property. 
Example: A PAC-field that is RG-Hilbertian but not Hilbertian. Let $G_{1}, G_{2}, G_{3}, \ldots$ be a listing that includes each nontrivial finite group just once (up to isomorphism), and suppose $\left|G_{1}\right|=2$. The profinite group $H=\prod_{i=1}^{\infty} G_{i}$ has countably infinite rank [FrJ, Ex. 15.13]. Hence $H$ is a quotient of $\hat{F}_{\omega}$ [FrJ, Cor. 15.20]. As in the proof of Corollary 2 it follows that the universal frattini cover $\tilde{H}$ of $H$ is also a quotient of $\hat{F}_{\omega}$. Since $\tilde{H}$ is projective, it embeds as a subgroup of $\hat{F}_{\omega}$.

Let again be $P$ a countable Hilbertian PAC-field. Then $\tilde{H}$ embeds into $G_{P} \cong \hat{F}_{\omega}$. Let $K$ be the fixed field of $\tilde{H}$ in $\bar{P}$. Then $K$ is PAC (since it is an algebraic extension of the PAC-field $P$ ) and every finite group is a Galois group over $K$. Hence $K$ is RG-Hilbertian by Theorem B. We now show that $K$ is not Hilbertian.

Let $\lambda: \tilde{H} \rightarrow H$ be the natural map. Then $\lambda^{-1}\left(G_{1}\right)$ is an extension of the group $G_{1}$ (of order 2) by the kernel of $\lambda$, which is pro-nilpotent [FrJ, Lemma 20.2]. Hence each surjection from $\tilde{H}$ to the symmetric group $S_{5}$ maps $\lambda^{-1}\left(G_{1}\right)$ to a solvable normal subgroup of $S_{5}$, which must be trivial. Thus the map $\tilde{H} \rightarrow G_{1}$ that is the composition of $\lambda$ with projection to $G_{1}$ does not factor through a surjection $\tilde{H} \rightarrow S_{5}$. This means that not all finite embedding problems over $K$ are solvable. Hence $K$ is not Hilbertian (by Theorem A).

\section{Bibliography}

[Ax] J. Ax, The elementary theory of finite fields, Annals of Math. 88 (1968), 239-271.

[Fr] M. Fried, On the Sprindžuk-Weissauer approach to universal Hilbert subsets, Israel J. Math 51 (1985), 347-363.

[FrJ] M. Fried and M. Jarden, Field Arithmetic, Springer-Ergebnisse der Math. und ihrer Grenz. 11 (1986).

[FrJ, 2] M. Fried and M. Jarden, Diophantine properties of subfields of $\bar{Q}$, Amer. J. Math. 100 (1978), 653-666.

[FrVo] M. Fried and H. Völklein, The inverse Galois problem and rational points on moduli spaces, Math. Annalen 290 (1991), 771-800.

[Fy] G. Frey, Pseudo algebraically closed fields with non-archimedean real valuations, J. of Algebra 26 (1973), 202-207.

[Hu] B. Huppert, Endliche Gruppen I, Springer, New York-Heidelberg-Berlin 1967

[Iw] K. Iwasawa, On solvable extensions of algebraic number fields, Annals of Math. 58 (1953), 548-572.

[JaLu] M. Jarden and A. Lubotzky, A transfer principle between the Hilbertian and the $\omega$-free properties, J. London Math. Soc., to appear. 
[Ka] G. Karpilovsky, Projective representations of finite groups, M. Dekker, New York and Basel 1985.

[LD] A. Lubotzky and L. v. d. Dries, Subgroups of free profinite groups and large subfields of $\bar{Q}$, Israel J. Math. 39 (1981), 25-45.

[Ma] H. Matzat, Konstruktive Galoistheorie, Lecture Notes in Math, Springer-Verlag 1284 (1986).

[M] O.V. Mel'nikov, Normal subgroups of free profinite groups, Math. USSR Izvestija 12 (1978), 1-20.

[P F. Pop, The theory of totally $\Sigma$-adic numbers, preprint 1990.

[Se] J.-P. Serre, Topics in Galois Theory, Research Notes in Mathematics, Jones and Barlett 1 (1992).

[U] K. Uchida, Separably Hilbertian fields, Kodai Math. Journal 3 (1980), 83-95.

[W] A. Weil, The field of definition of a variety, Amer. J. Math 78 (1956), 509-524.

[Ws] R. Weissauer, Der Hilbertsche Irreduzibilitätssatz, J. für die reine und angew. Math. 334 (1982), 203-220.

Mike Fried

Department of Mathematics

UC Irvine

Irvine, California 92717
Helmut Völklein

Department of Mathematics

University of Florida

Gainesville, Fl 32611 